\title{
Application of Cloud Computing Technology on Computer Secure Storage
}

\author{
You Zhou, Yu Tang \\ Jiangxi Modern Polytechnic College, Nanchang, Jiangxi, 330095
}

\begin{abstract}
Keywords: Cloud Computing, Computer Secure Storage, Application Method
\end{abstract}
\begin{abstract}
In the big data era, how to effectively store, analyze and effectively use massive data information? This is the current need of our social and economic development. The cloud computing technology has changed the traditional host era, which has more superb computing power, analysis capabilities and storage capabilities, the Internet technology and computer technology to effectively combine to trigger the transformation of computer information technology. At the same time, with the development of network information age, the security of information is getting more and more attention from everyone. This article systematically explains the concept and characteristics of cloud computing technology, computer network security issues and how cloud computing technology is used in computer network security storage for reference.
\end{abstract}

\section{Introduction}

Cloud computing is an Internet-based service related to the increase, use and delivery models that are generally used for the Internet to dynamically scale and provide virtual resources. Cloud computing is more capable than traditional computer computing, and it reaches 10 trillion operations per second. Therefore, it is widely used to predict weather changes, simulate nuclear explosions and market trends. Users only need to use mobile phones and computers to connect to the data center to calculate according to their needs. At present, there are three types of cloud computing services: IaaS: Infrastructure as a service, users can get perfect computer infrastructure services through the Internet, such as renting hardware servers; PaaS: Platform as a Service is actually a software development platform as a Sass: Software as a Service: Cloud computing developers provide software over the Internet, users do not have to buy software, just rent software from vendors. In the past two years, cloud computing has developed rapidly. Many network operators are actively developing cloud services, and the market for private cloud services is also expanding. In 2015, the market size of cloud services reached 52.24 billion U.S. dollars, up 20.6\%. Expected to grow in the next few years. The difference between cloud computing and traditional computers is that they are transmitted over the Internet. They are calculated on a large number of distributed computers rather than on local computers or remote servers. Its data center, like the Internet, can be stored and calculated on demand by enterprises or individuals simply by switching resources to the desired application. It is very large scale, low cost, easy to access, virtualization, reliability, versatility, scalability and potentially huge risk characteristics.

\section{Computer Network Security Issues}

With the development of computer and mobile internet, the problem of network security is becoming more and more important. In recent years, many international cyber security incidents have been launched by international hacker organizations and cyber terrorist organizations. In May 2015, the information of 100,000 taxpayers in the United States was stolen, resulting in a direct economic loss of 50 million U.S. dollars. In June, the Japanese government's pension information system was hacked, revealing 1.25 million personal information; in October, the British telecom operator Talk talk was hacked, resulting in the disclosure of 4 million user information. This shows that computer network security is related to personal, business, social and national information security. At present, under the wave of Internet economy, various computer softwares and mobile phone APPs emerge in an endless stream, and the speed of upgrading is very fast. Some of these 
software and mobile phone APP itself has many flaws but it is recommended to install and use. Therefore, in the course of using the network security problems often appear. In the meantime, all kinds of computer virus and mobile phone virus are sent to the user through SMS, telephone, etc., and the user is infected without the knowledge. Computer virus is a special symbol, it can quickly copy and reproduce, it can spread a large area, with serious consequences. Network hackers use viruses to intrude on personal computers or mobile phones, thereby obtaining user's personal information such as personal shopping information, bank card password, and then silently transferring the property in the user's account to cause serious losses to the user. The cloud computing database through encryption technology, only the owner of the data through the password to enter the data center, for other cloud computing users is to implement the confidentiality. But there is no secret to cloud providers. In recent years, while vigorously developing cloud computing, our country has also stepped up the security management of Internet security and cloud computing technologies. On March 29, 2012, the Ministry of Industry and Information Technology, the Ministry of Science and Education, and the National Natural Science Foundation jointly formulated the Opinions on the Development and Construction of the Next Generation Internet "The 12th Five-year Plan". On March 4, 2014, at the China International Cloud Computing Technology and Applications Exhibition, the relevant person in charge of the Ministry of Industry and Information Technology revealed that the draft cloud computing standardization technology system has been formed. In addition, important progress has been made in the global research and development of cloud computing security. Such as China to create IBM cloud computing center, Kaspersky and Rising foreign companies introduced network security solutions, there have been Microsoft cloud, Google cloud, will continue to promote the security of cloud computing applications.

\section{Cloud Computing Technology for Network Security Storage Key Technologies}

Password verification. The system creates a valid user name password for the user in need. During the authentication process, the user only needs to input the corresponding user name and password according to the prompt, that is, the user can perform the verification and the verification is correct and the user is deemed as the valid user, otherwise, unauthorized user. smart IC card application authentication. Enter the user's legitimate information into the smart IC card, enter the user name and password when the user needs authentication, the smart card can be sent to the authentication server random number for verification, greatly enhancing the network's security performance. Kerberos authentication. This is a third-party trusted authentication protocol under the authentication method, it has a resource access mechanism and authorization server, the user's password can generate a key $\mathrm{K}$, and then through the authorization server ticket for authentication, access to the system After the legal certificate, you can get the appropriate service. PKI authentication. This is based on the security certification under the public key infrastructure. It implements the encryption and decryption process through the key matching each other, and realizes the security service under the key backup and recovery mechanism and key update mechanism.

Data Encryption Data Encryption mainly consists of symmetric encryption algorithms and asymmetric encryption algorithms. Among them, the symmetric encryption algorithm has higher encryption and decryption speed and higher confidentiality. However, it is more difficult to transmit and manage the keys , But also the lack of signatures. The asymmetric encryption algorithm makes up for the shortcomings of the symmetric encryption algorithm, which is simpler for key delivery and management efficiency, with higher security, however, this encryption, decryption slower and more complex .

Cloud computing data center storage disaster recovery and recovery technology to preserve the integrity and availability of data, including the scalable technology through the scalable large-capacity storage pool to manage the storage of physical space, it completely changed the management of the file system Way, is no longer limited to a certain physical equipment, greatly enhanced the security performance of storage systems. 
Among the cloud computing secure storage platforms, managing and sharing files is a common problem, which is a key sharing and key management problem. In the distributed file security storage system of a computer, the key management Secure storage services enable efficient management and service.

In the distributed storage system of computer network, because the location of error information is uncertain and unknown, erasure code technology can be used. Its elements mainly include information symbols, supervision symbols, code words, code sets, block codes And other information, three different types of erasure code technologies can be used: RS erasure code; no rate encoding; concatenated low density erasure codes, which can greatly improve the speed of encoding and decoding and improve the reliability and quality of computer networks .

\section{Cloud Computing Technology in Computer Network Security Storage Application}

This is a computer algorithm under the challenge-response-authentication mechanism. It introduces the concept of error-correcting coding. When a user of cloud computing queries the data, he verifies the status of the data and challenges the cloud. According to the user's The challenge is to respond. At this time, the user determines the security status of the cloud data through the response of the computer. If the verification fails, it proves that the data center file may be damaged and needs to be repaired. If the damage value is within the threshold, then You can use the code to restore the original data. If the verification is passed, the user can directly enter the cloud computing or storage. In the process of network computer running, often due to the failure of the computer itself caused by data corruption, coding in the recovery of data information has a relatively strong advantage. Through this challenge, response, validation algorithm that allows users to verify the integrity of the cloud database and security, but also to achieve precise positioning and analysis of cloud errors.

In order to ensure the safety of data stored in the cloud, MC-R, MC-R in the cloud and MC-R policy of the policy user can generally be adopted to achieve management and security control over user data. The application of MC-R strategy is mainly reflected in two aspects: one is the encryption calculation of the client MC. Due to the insecure network environment, data hiding and data masquerading performance are relatively poor. In particular, some hackers on the network can exploit programs or client vulnerabilities to invade the data center and cause data Information leakage and so on. Therefore, in order to improve the security of network computer cloud storage, the database and the cloud are generally encrypted. The client's MC encryption method can realize the construction of data hiding module, data masquerading module and data marking module. The three modules each have their own advantages, and the three are in a state of synergy with each other to realize the safe storage function of the cloud computing technology. On the other hand, they are cloud-based RSA applications. Cloud computing has supercomputing power, so it only needs to encrypt the core data and key data in practice, so it can effectively avoid the heavy data consumption status of the cloud RSA. Its encryption and decryption module construction, mainly is: First, the user's needs to build a set of RSA company key, and then save the user authored by MC encryption algorithm to process the data, upload to the cloud, the cloud then encrypt the data . Finally, the cloud users to download encrypted files, unlocked by the key to find the hidden data, you can restore the data at the beginning of the state.

\section{Conclusion}

Cloud computing is a new business computing model. Using high-speed Internet transmission capabilities, the data processing process from the personal computer or server to the Internet cluster of computers. As the core technology of next-generation network computing platform, it provides reliable and secure data storage, convenient and efficient Internet services and powerful computing capabilities. With the development of the Internet, computer network security is more and more important. It is necessary to apply cloud computing in computer network security storage and improve the security of cloud computing storage. 


\section{References}

[1] Fang Bing, Xiao Pengfei.Analysis of the color of the web design art [J]. Computer fans. 2017 (09)

[2] Zhao Lu, Zou Di.J] .Network Security Technology and Application. 2017 (10)

[3] Zhao Xin. Cloud computing technology in China's rural information technology in the application of research [J]. Rural Technology. 2017 (19)

[4] Song Rong. Analysis of Cloud Computing Technology Based on Intelligent Cloud Network Architecture [J]. Computer Fans. 2017 (10)

[5] Gao Xiang. Cloud Computing Technology Development Analysis and Application [J]. China New Technology and New Products. 2016 (13)

[6] Gan Lu, Sun Junju.Network security defense technology under cloud computing technology [J] .Network Security Technology and Application. 2016 (02) 